Supporting Information

Tribo-Induced Smart Reflector for Ultrasensitive Self-Powered Wireless Sensing of Air Flow Jiaqi Wang ${ }^{\dagger,}$, Pengcheng Liu ${ }^{\S}$, Cuiling Meng§, Hoi Sing Kwok ${ }^{\S}$, Yunlong ZZi ${ }^{*}$,

†. School of Marine Sciences, Sun Yat-Sen University, Zhuhai 519082, PR China.

¥ Department of Mechanical and Automation Engineering, The Chinese University of Hong Kong, Shatin, N.T., Hong Kong, China.

\$. State Key Laboratory on Advanced Displays and Optoelectronics Technologies, Hong Kong University of Science and Technology, Clear Water Bay, Kowloon, Hong Kong, China.

Email: ylzi@cuhk.edu.hk

1. Fabrication process of the developed tribo-induced smart reflector: As shown in Figure S1, to fabricate the reflector, two $1.13 \mathrm{~mm}$ thick transparent glasses with the size of $15 \mathrm{~mm}$ by $20 \mathrm{~mm}$ were firstly prepared. Indium tin oxide (ITO) was coated on the one surface for both two glasses, serving as the transparent electrodes. Afterwards, chemical etching was performed for the two ITO electrodes. For the two glasses, the ITO electrode was patterned with a $2 \mathrm{~mm}$ by $2 \mathrm{~mm}$ active area and a line-shaped electrical connector. Such the miniature active region guarantee that the developed smart reflector can be actuated by the ultralow charge loading. For one of the two glasses, the glass surface opposite to the ITO electrode was coated with a silver layer, functioning as the reflector. As described in the Method section, the alignment layer was prepared before spincoated on the two glasses. As for the LC-based devices, the alignment layer has the capability to achieve the orientation control of the LC molecules supplying a sufficient anchoring energy. The alignment layer in this work provides the LCs with an excellent and uniform vertical alignment. The vertically aligned configuration empowers the PNLC normally transparent property since the LC and LC monomer have the identical refractive index in the short-axis. The phase-separation was performed to achieve the vertical alignment, where the alignment layer was cooling treated. During the cooling process, the LC monomer was separated from the polyimide since its interaction strength with the polyimide is greatly alleviated, leaving the vertically-aligned LC monomer. After the phase separation, the morphology of the alignment layer was greatly changed, evolving from a smooth surface to a puddle-like inhomogeneous surface. To tighten the LC monomer and the alignment layer and to maintain the puddle-shaped surface morphology, the layer was situated under the ultraviolet (UV) irradiation for $2 \mathrm{~min}$, where the LC monomer was partially cross-linked. After that, the two glasses embedded with the treated alignment layers were positioned to face each other, where the $2 \mathrm{~mm}$ by $2 \mathrm{~mm}$ active areas of two glasses were precisely aligned to overlap with each other. $8 \mu \mathrm{m}$ gap was formed between the two glasses, attributed to the assistance of silicon dioxide spacers. The gap was then encapsulated by a mixture of a fluidic 
nematic LC and the LC monomer. It should be noticed that upon the UV light irradiation, the nematic LC cannot be cross-linked. Thanks to the long alkyl side chain of the alignment layer, both nematic LC and LC monomer were homeotropically aligned. Therefore, the LC bulk presented a transparent property when no charge was loaded. At the final stage, the encapsulated cell was illuminated by the UV light once again. Upon illuminated by the UV light, the LC monomer began to cross link with the other LC monomers inside the encapsulated mixture or embedded in the alignment layers. As a result, the LC based polymer network inside the fluidic nematic LC continuum was achieved, leaving a PNLC based tribo-induced smart reflector.

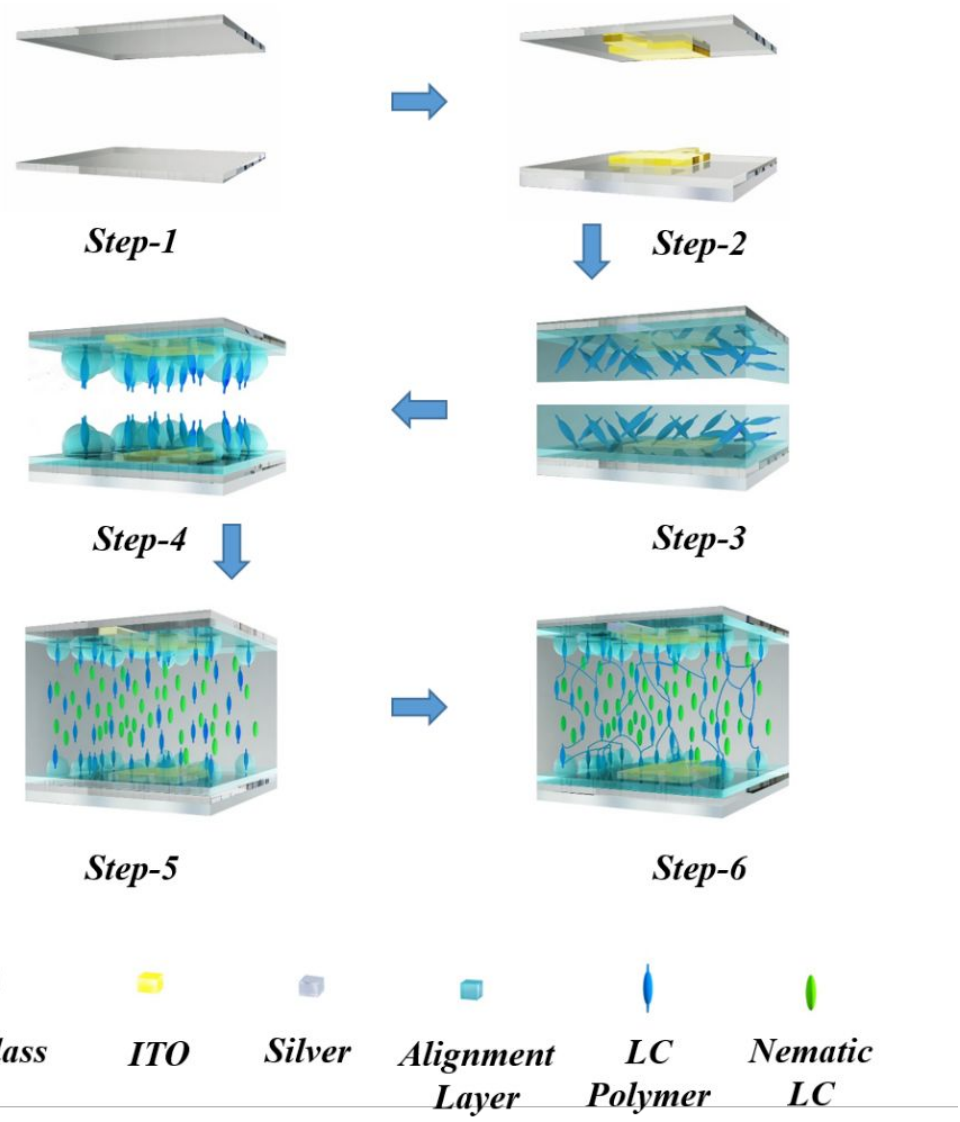

Figure S1. Fabrication process of the tribo-induced smart reflector. 
2. Measured transmittance and haze ratio of the polymer network liquid crystal (PNLC) without the silver coated: To evaluate the optical performance of the PNLC, a PNLC with the active region of $10 \mathrm{~mm}$ by $10 \mathrm{~mm}$ was prototyped. The fabricated PNLC was then powered by a commercial voltage source in alternating current (AC) mode. The frequency for powering is 1000 $\mathrm{Hz}$. The voltage drop on the PNLC was tuned from $0 \mathrm{~V}$ to $60 \mathrm{~V}$, and the transmittance and haze ratio measurements were performed, the results of which is indicated in Figure S2.
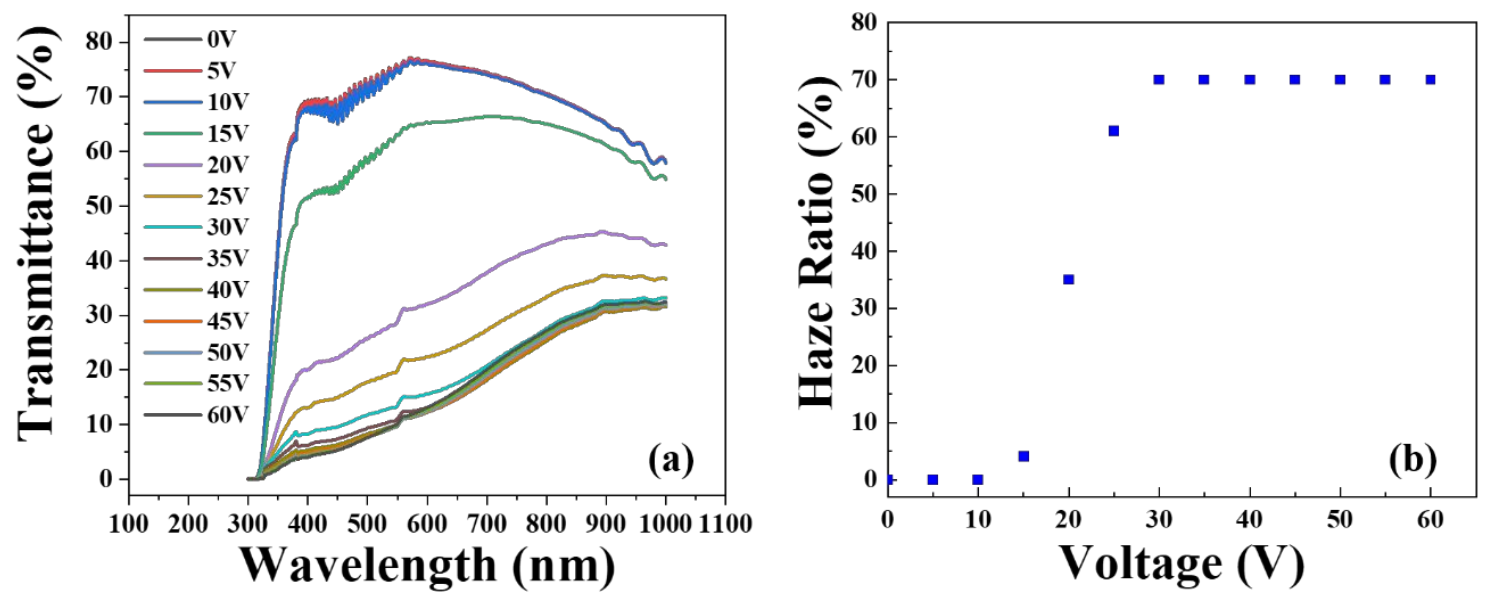

Figure S2. The dependence of measured transmittance and haze ratio on the voltage drop of the PNLC without silver coated under the driving of commercial voltage source in $1000 \mathrm{~Hz}$ AC mode. (a) Transmittance. (b) Haze ratio.

3. The time-variant transferred charge amount of the aerodynamically-driven TENG under the short-circuit (SC) condition under the various wind speeds: Employing the Keithley 6514 electrometer as the coulomb meter, the profile of the SC transferred charge was measured under the different wind speeds loaded on the TENG, the results of which is shown in Figure S3. 

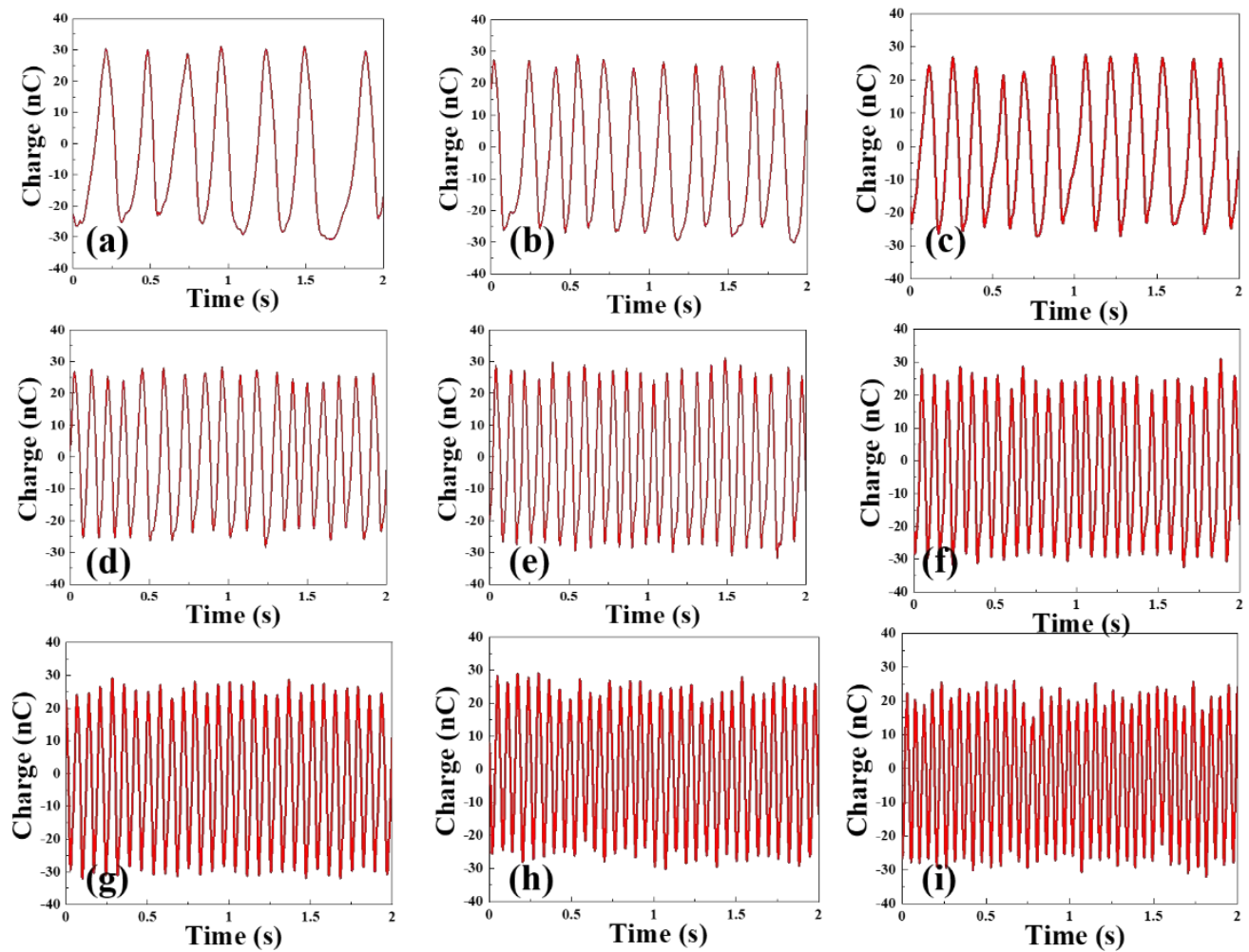

Figure S3. The time-variant transferred charge amount of the aerodynamically-driven TENG under the short-circuit (SC) condition under the various wind speeds. From a-i, the wind speed loaded is $2.5 \mathrm{~m} / \mathrm{s}, 3.5 \mathrm{~m} / \mathrm{s}, 4 \mathrm{~m} / \mathrm{s}, 5.5 \mathrm{~m} / \mathrm{s}, 6.7 \mathrm{~m} / \mathrm{s}, 7.5 \mathrm{~m} / \mathrm{s}, 8.1 \mathrm{~m} / \mathrm{s}, 9 \mathrm{~m} / \mathrm{s}$ and $10 \mathrm{~m} / \mathrm{s}$.

4. Fast Fourier transformation (FFT) results of the time-variant SC transferred charge amount of the TENG: Based on the time-variant SC transferred charge amount as indicated in Figure S3, FFT was performed and the results are shown in Figure S4. 

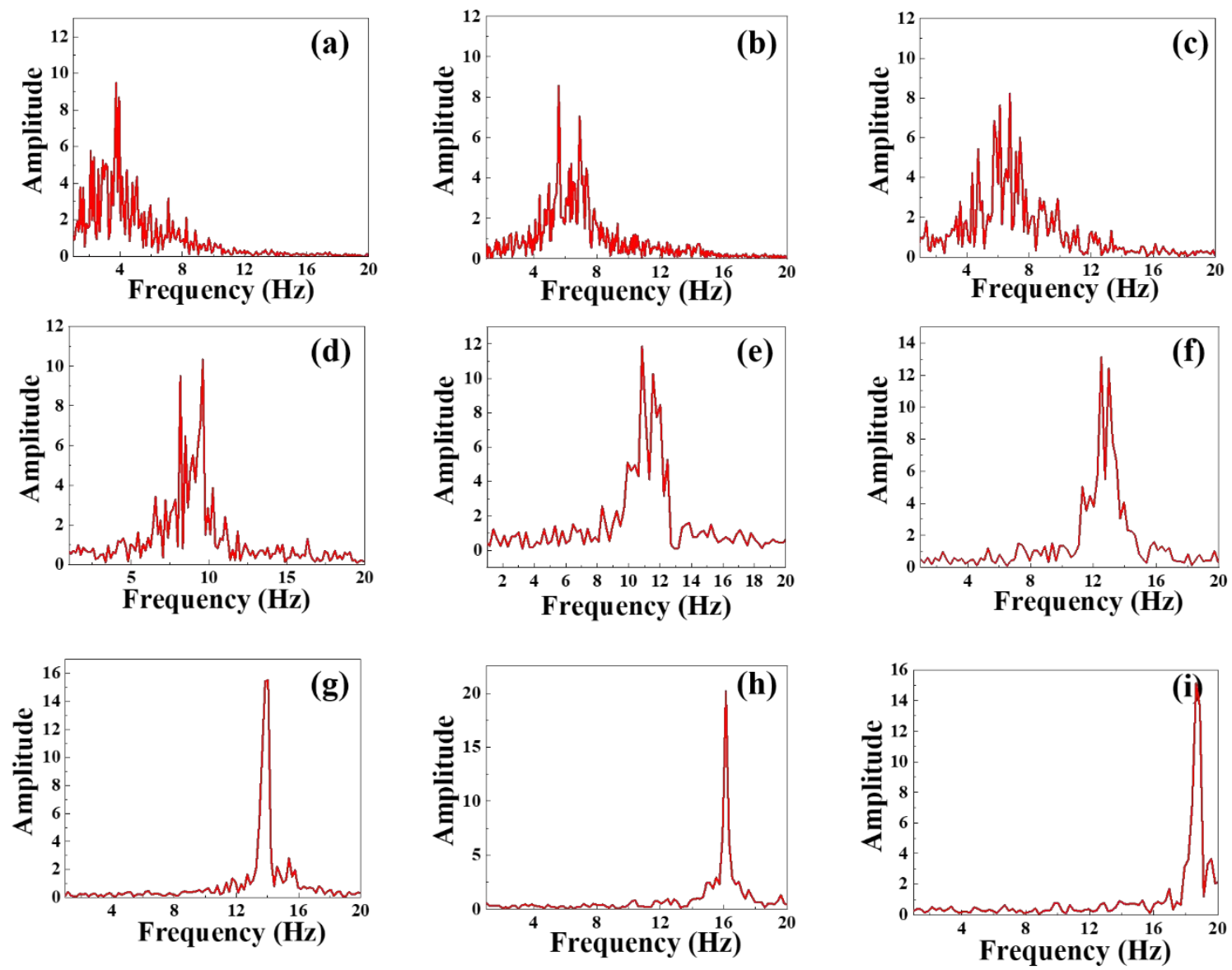

Figure S4. The FFT results of the time-variant SC transferred charge mount under the various wind speed loaded. From a-i, the wind speed loaded is $2.5 \mathrm{~m} / \mathrm{s}, 3.5 \mathrm{~m} / \mathrm{s}, 4 \mathrm{~m} / \mathrm{s}, 5.5 \mathrm{~m} / \mathrm{s}, 6.7$ $\mathrm{m} / \mathrm{s}, 7.5 \mathrm{~m} / \mathrm{s}, 8.1 \mathrm{~m} / \mathrm{s}, 9 \mathrm{~m} / \mathrm{s}$ and $10 \mathrm{~m} / \mathrm{s}$.

5. Electrical characterization circuit of the tribo-induced smart reflector: as illustrated in Figure S5, a measurement circuit was constructed to reveal the relationship between the voltage drop on the PNLC based tribo-induced smart reflector $V$ and the amount of transferred charge $Q$. 


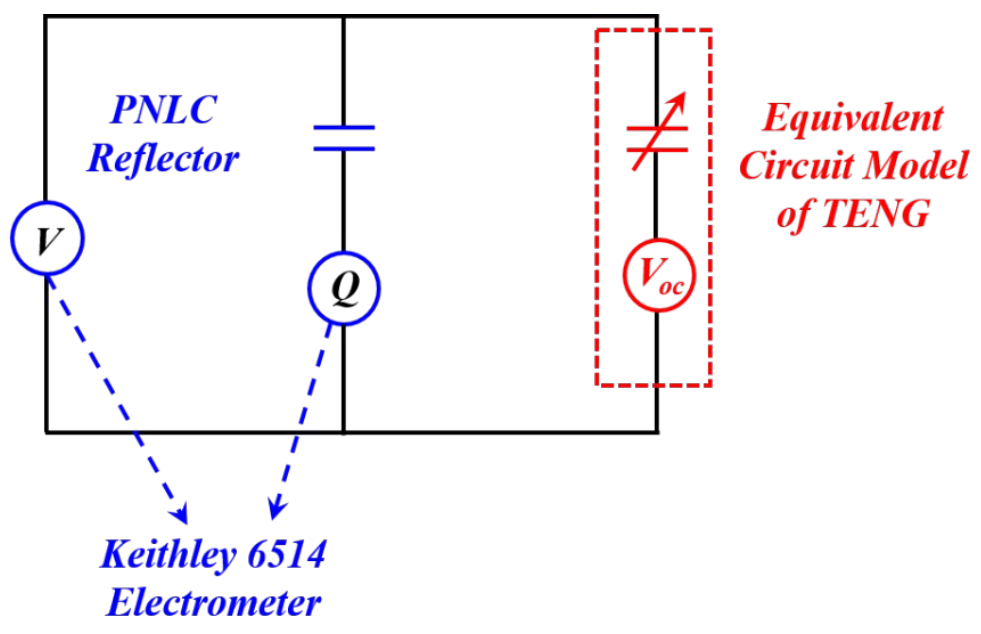

Figure S5. Constructed electrical characterization circuit to investigate the relationship between the voltage drop on the PNLC based tribo-induced smart reflector and the amount of transferred charge.

\section{Supporting Videos}

Video S1: The flutter effect of the aerodynamically-driven TENG (the video is played in 10 times slower speed) (MP4)

Video S2: The appearance variation of the smart reflector before and after tribo-charging by the wind-driven TENG (MP4)

Video S3: The reflectance mode switching of the smart reflector upon the tribo-charging by the wind-driven TENG (MP4)

Video S4: The optical behavior variation of the smart reflector upon the TENG was driven by the expiration (MP4) 